\title{
Evaluation of the Efficacy of Vitamin D Supplementation With Two Different Doses During Pregnancy on Maternal and Cord Blood Vitamin D Status, Metabolic, Inflammatory and Oxidative Stress Biomarkers, and Maternal and Neonatal Outcomes: a Study Protocol
}

\author{
Soudabe Motamed ${ }^{1}$, Bahareh Nikooyeh ${ }^{2}$, Tirang R. Neyestani* ${ }^{2}$ \\ 1- PhD Student in Nutrition Sciences, National Nutrition and Food Technology Research Institute, Faculty of Nutrition Sciences and Food Technology, \\ Shahid Beheshti University of Medical Sciences, Tehran, Iran \\ 2- Laboratory of Nutrition Research, National Nutrition and Food Technology Research Institute, Faculty of Nutrition Sciences and Food Technology, \\ Shahid Beheshti University of Medical Sciences, Tehran, Iran
}

\section{A B S T R A C T}

Background and Objectives: Vitamin D deficiency during pregnancy is prevalent throughout the world and has been associated with complications in mothers and infants. The aim of this study is to evaluate the efficacy of two doses of vitamin D supplementation (1000 IU/d versus $2000 \mathrm{IU} / \mathrm{d}$ ) during pregnancy on maternal and cord blood vitamin D status, metabolic, inflammatory and oxidative stress biomarkers as well as maternal and neonatal outcomes (birth size and Apgar score).

Methods: A total of 84 pregnant women with the eligible criteria are randomly allocated to one of the two supplementation groups: (a) $1000 \mathrm{IU} / \mathrm{d}$ vitamin D which is routinely given to pregnant women, and (b) $2000 \mathrm{IU} / \mathrm{d}$. Biochemical assessments of mothers are performed at the beginning and 34 weeks of gestation. They include serum concentrations of 25-hydroxycalciferol (25(OH)D), calcium, phosphate, intact parathyroid hormone (iPTH), fasting serum glucose (FSG), insulin, lipid profile including triglycerides (TG), total cholesterol (TC), low-density lipoproteincholesterol (LDL-C) and high-density lipoprotein cholesterol (HDL_C), as well as high sensitivity C-reactive protein (hsCRP) and the maternal cell-culture supernatant concentrations of inflammatory cytokines IL- $1 \beta$, IL- 6 and TNF- $\alpha$. Assessments of infants at delivery comprise cord blood serum concentrations of 25(OH)D, iPTH, hs-CRP, IL-1 $\beta$, IL-6, TNF- $\alpha$ birth sizes and Apgar score.

Discussion: The results obtained from the clinical efficacy trials on the appropriate dose for vitamin D supplementation during pregnancy are controversial. The current study could be a step to attenuate the existent controversy on evaluating the efficacy of two doses of vitamin D supplement on a wide spectrum of both mother and newborn outcomes. Meanwhile our study could give a clue to the policy-makers at $\mathrm{MOH}$ by revealing the efficacy of currently prescribed $1000 \mathrm{IU} / \mathrm{d}$ vitaminD3 versus $2000 \mathrm{IU} / \mathrm{d}$.

Keywords: Vitamin D supplementation, Clinical efficacy trial, Pregnancy, Study protocol

\section{Introduction}

Vitamin D deficiency or insufficiency is widespread throughout the world in almost all ages and sex subgroups, including pregnant women (1, 2).The prevalence of vitamin $D$ deficiency among Iranian pregnant women has been reported to range from 60 to $80 \%(3,4)$. Because of the physiological changes that occur during pregnancy, pregnant women are considered to be the most vulnerable groups for vitamin D deficiency and insufficiency, even in regions with abundant sun exposure (5-8).

Vitamin D deficiency might affect both mothers and their neonates, and result in several adverse maternal and neonatal outcomes including gestational diabetes, preeclampsia, preterm delivery, abortion, cesarean, impaired fetal skeletal development, small 
for gestational age (SGA), low birth weight (LBW), newborn's wheezing and asthma (9-13).

Vitamin $D$ as an immune modulator has an important role in maternal tolerance to the fetus and correctly embryonic implantation (14-18). Indeed, it has been suggested that the regulatory effect of vitamin D on immune function and inflammation is its primary role during pregnancy. Therefore, it seems vitamin D deficiency has modulatory effects on immune function (both innate and adaptive immunity), inflammatory reactions and oxidative stress, which is associated with the presence of aforementioned adverse pregnancy outcomes (19-21).

The results of several studies have shown that vitamin $\mathrm{D}$ requirement during pregnancy exceeds the recommended value by the Institute of Medicine (IOM; 600 IU/d) (22) to achieve the serum concentrations of $>75 \mathrm{nmol} / \mathrm{L}$ which is considered sufficient level for all individuals regardless of pregnancy status (23). In addition, it has been suggested that vitamin D supplementation during pregnancy might reduce the risk of maternal comorbidities attributed to vitamin D deficiency or insufficiency and may help improve neonatal outcomes (24-26). However, there are still inconsistencies in the results of trials and considerable controversy surrounding the definition of the cut-off values for vitamin D deficiency and insufficiency during pregnancy $(27,28)$. As a result, previous interventional studies failed to determine an appropriate (safe and effective)doseof vitamin D supplementation for pregnant women.

Therefore, the primary objective of this study wasto evaluate the safety and efficacyof $1000 \mathrm{IU} / \mathrm{d}$ vitamin D3 (that is approved to be administered to Iranian pregnant women) compared with $2000 \mathrm{IU} / \mathrm{d}$ vitamin D3 in providing sufficient level of $25(\mathrm{OH}) \mathrm{D}$ in mothers and cord blood. The secondary objective was to investigate the effects of vitamin D supplementation (both $1000 \mathrm{IU} / \mathrm{d}$ and $2000 \mathrm{IU} / \mathrm{d}$ ) on the metabolic, inflammatory and oxidative stress biomarkers of maternal and cord blood serum, as well as, neonatal outcomes (weight, length, head circumference andApgar score).

Primary objectives: Evaluation and comparison of the efficacy of 1000 vs.2000 IU/d vitamin D supplementation during pregnancy on maternal and newborn vitamin D status.

Secondary objectives: Evaluation and comparison of the efficacy of $1000 \mathrm{vs.2000} \mathrm{IU/d} \mathrm{vitamin} \mathrm{D}$ supplementation during pregnancy on maternal and cord blood metabolic, inflammatory and oxidative stress biomarkers as well as neonatal outcomes (birth sizes and Apgar score).

Hypothesis: We hypothesize that taking $2000 \mathrm{IU} / \mathrm{d}$ vitamin $\mathrm{D}$ isa more effective dose than $1000 \mathrm{IU} / \mathrm{d}$ during pregnancy in providing optimal levels of vitamin $\mathrm{D}$ and metabolic, oxidative stress and inflammatory biomarkers as well as more desirable birth outcomes.

\section{Subjects and Methods}

Study design: A double-blind randomized clinical trial is designed among pregnant women attending the outpatient obstetric clinics of three hospitals (with similar culture, education and economic status) in Tehran between February 2017 and January 2018.

During this period of time, pregnant women who refer to the obstetric clinics for prenatal care during the first trimester of gestation at the three hospitals are assessed for eligibility. Eligible subjects are invited to the study and those who are willing to participate by using block randomization are randomly allocated to one of the two vitamin D supplementation groups: (a) $1000 \mathrm{IU} / \mathrm{d}$; and (b) 2000 IU/d. Figure 1 outlines the details. A $1000 \mathrm{IU} / \mathrm{d}$ arm is chosen rather than placebo or the recommended RDA $(600 \mathrm{IU} / \mathrm{d})$, as it is a routine protocol for vitamin D supplementation during pregnancy in Iran. Obviously, it is unethical to give placebos to the pregnant women when there is an official direction guide for vitamin $\mathrm{D}$ supplementation during pregnancy from $\mathrm{MOH}$.

Calculation of sample size: We calculated a sample of 37 subjects in each group with assuming an effect size of 0.75 and the power of $90 \%$.

Both intervention groups could receive supplements commonly prescribed during pregnancy including multivitamin containing 400 IU vitamin D3 or less, ferrous sulfate and folic acid. The vitamin D3 tablets are manufactured by Jalinous Company, Tehran, Iran. 


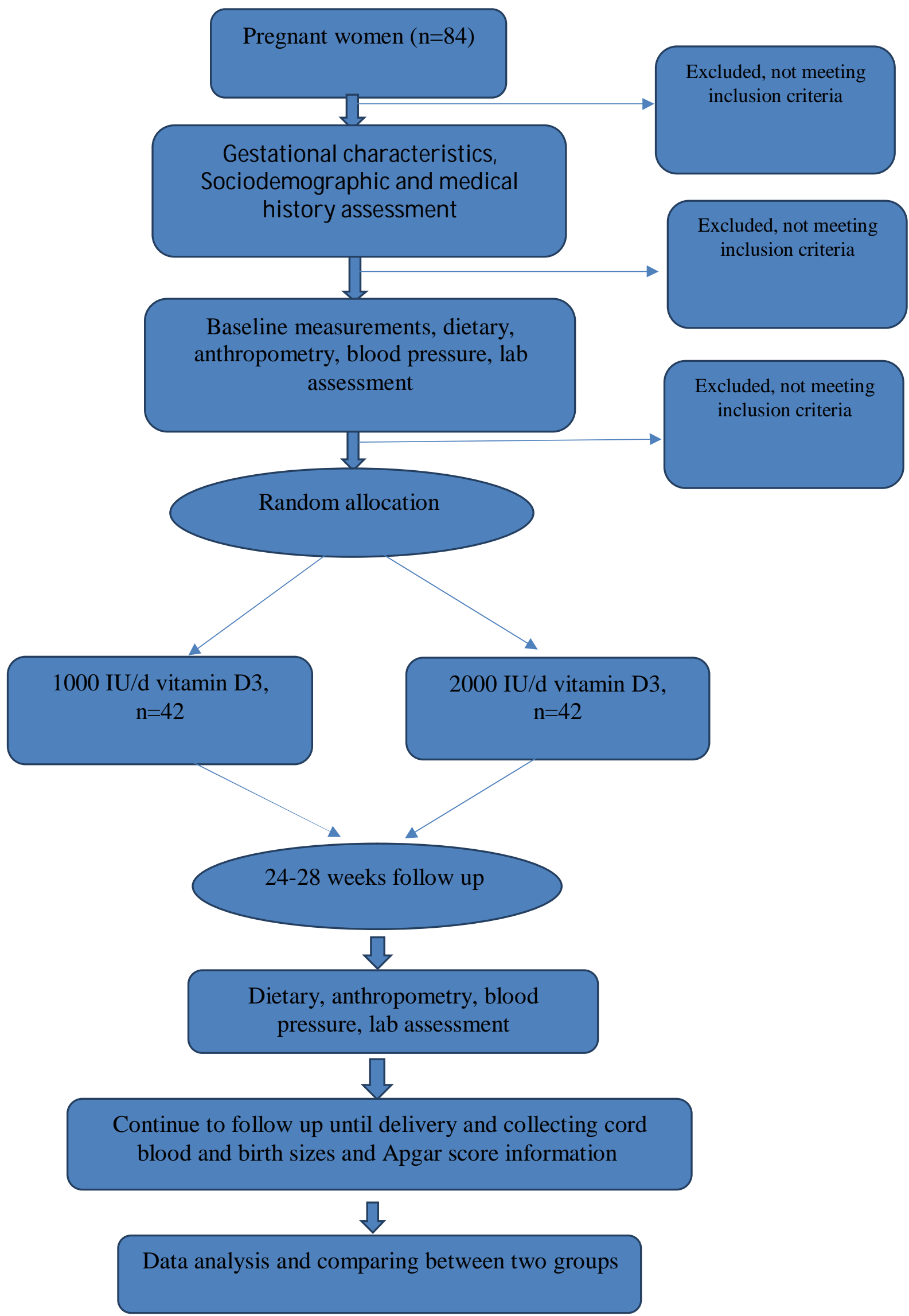

Figure 1. A summary of interventional study 
Inclusion criteria: The inclusion criteria for participation are maternal age of 18-40 years, gestational age of 12 weeks or less (gestational age is calculated based on the first day of the last menstrual period (LMP) for women with regular cycles or ultrasonography result is considered for women who are unsure about their LMP or have irregular cycles), singleton pregnancies, mothers supposed to have normal pregnancy and not having preexisting diseases like hypertension, or cardiac, renal, hepatic, autoimmune, rheumatoid arthritis and digestive disease, or endocrinological disorders including diabetes (type 1 or type 2 diabetes), parathyroid disorders, and thyroid disorder, albeit mothers who underwent treatment by drug and have normal level of thyroid hormones could participate in the study, not receiving dietary supplements including vitamin D (>600 IU/d), or omega-3 within the past 3 months before the intervention, not taking medications that could potentially influence vitamin D metabolism (notably estrogens and calcitonin), steroidal antiinflammatory or anticoagulant medications and willingness to participate in the study.

Exclusion criteria: Participants who do not meet the inclusion criteria, diagnosed to have fasting blood sugar (FBS) $>92$ at first blood sampling, or blood pressure $>140 / 90 \mathrm{mmHg}$ at the first visit, use extra vitamin D3 supplement in addition to what is administered (protocol deviation), omega-3 or other medications that could potentially influence vitamin D metabolism (notably estrogens and calcitonin), steroidal anti-inflammatory or anticoagulant medications during the intervention, fetal anomaly, poor adherence to the study protocol and unwillingness to continue the intervention.

Primary outcomes are changes in maternal serum 25(OH)D concentration from the first trimester till 34-36 weeks of gestation. Based on the Endocrine Society Practice Guideline and Institute of Medicine (IOM) for adults, vitamin D status was categorized as: (1) deficiency ( $<50 \mathrm{nmol} / \mathrm{L})$, (2) moderate deficiency, $(50-75 \mathrm{nmol} / \mathrm{L})$, and (3) normal status (>75 nmol/L) (29).

Secondary outcomes are changes in maternal cell culture supernatant concentrations of inflammatory cytokines including Interleukin 1 beta (IL-1 ), Interleukin 6 (IL-6) and tumor necrosis factor alpha (TNF- $\alpha$ ) and changes in maternal serum concentrations of high sensitivity C-reactive protein (hs-CRP), intact parathyroid hormone (iPTH), calcium, phosphate, glycemic status (glucose, insulin, homeostatic model assessment of insulin resistance(HOMA-IR)), lipid profile (triglyceride (TG), total cholesterol (TC), low-density lipoprotein cholesterol (LDL-C),high density lipoproteincholesterol (HDL-C)) and cord blood serum concentrations of $25(\mathrm{OH}) \mathrm{D}$, hs-CRP, IL-1 $\beta$, IL-6 and TNF- $\alpha$, as well as neonatal anthropometric measures including birth weight $(\mathrm{g})$, head circumference $(\mathrm{cm})$, length $(\mathrm{cm})$, APGAR score 1 and 5 minute $^{1}$. The other secondary outcomes are gestational diabetes defined as glucose intolerance detected for the first time during pregnancy based on the new guidelines for diagnosis of gestational diabetes ${ }^{2},(30)$, preterm delivery $^{3}(31)$, Low birth weight (LBW) (birth weight of infants lower than $2500 \mathrm{~g}$ (32)), miscarriage (pregnancy loss prior to 20 weeks of gestation (33)), preeclampsia diagnosed by a blood pressure of 140/90 $\mathrm{mmHg}$ or greater and the onset of 24-hour proteinuria $\geq 0.3 \mathrm{~g}$ at $>20$ weeks of gestation in women without previously hypertension (34) and type of delivery (cesarean section or vaginal delivery).

Data collection and variables: Two study visits are done at the first trimester and 34-36 week of gestation. At the first visit, socio-demographic information including age, educational status preexisting diseases, medical history, reproductive characteristics are collected from each participant after describing the objectives and the protocol of the study to her and taking an informed written consent. Moreover, information about duration of sun exposure (the number of minutes/hours that participants have spent in daylight) (35)and life style (dietary intake and physical activity level) are collected using questionnaires by a trained interviewer at the first and last visits.

Dietary intakes are assessed using 24-h recall questionnaire through which the quantity and type of both food and beverages consumed in the previous 24-hour period midnight to midnight on in two days of a week are evaluated. The analysis of 24-h recalls to measure the amount of nutrient intakes is done by using estimated values in household measurements

\footnotetext{
${ }^{1}$ APGAR score includes 5 components including appearance, pulse, grimace, activity, and respiration, each of which is given a score of 0-2 and reported at 1 and 5 minutes after birth.

${ }^{2}$ Fasting blood glucose level of $5.1 \mathrm{mmol} / \mathrm{L}(92 \mathrm{mg} / \mathrm{dL}), 1-\mathrm{h}$ postprandial glucose level of $10.0 \mathrm{mmol} / \mathrm{L}(180 \mathrm{mg} / \mathrm{dL})$ and/or a 2-h postprandial glucose level of $8.5 \mathrm{mmol} / \mathrm{L}(153 \mathrm{mg} / \mathrm{dL})$

${ }^{3}$ The onset of labor at less than 37 completed weeks of gestation
} 
and Nutritionist IV software (First Databank, San Bruno, CA, USA) modified for Iranian foods. Dietary intakes are not the main exposure variable and only will be assessed to adjust the probable differences between two groups.

Anthropometric measures and blood pressure: Anthropometric measures and blood pressure of all participants are measured at each antenatal visit. Height is measured only at the first visit. Body mass index (BMI) is calculated as weight in $\mathrm{kg}$ divided by height in meters squared.

Routine obstetrical visits are every 4 weeks until 28 weeks, every 2 weeks until 36 weeks, and then weekly until delivery. Information of doctor's visits, health status of participants, and medications is obtained from the mother's records.

Infant's birth weight $(\mathrm{g})$, length $(\mathrm{cm})$ and head circumferences $(\mathrm{cm})$ are recorded for each infant.

Laboratory investigations: After $12-14 \mathrm{~h}$ of fasting, maternal blood sample $(10 \mathrm{~mL} ; 5 \mathrm{~mL}$ in tubes without anticoagulant and $5 \mathrm{~mL}$ in heparinized falcon) and urine samples are collected at first (first trimester of gestation) and last visit (34-36 weeks of gestation). In addition, cord blood is obtained after clamping following delivery. Samples are sent to the Laboratory of Nutrition Research, NNFTRI, in cold box to separate serum and plasma by centrifugation at $1500 \mathrm{~g}$ for 10 minutes at $25 \mathrm{C}^{\circ}$. Serum and urine samples are kept in $-80^{\circ} \mathrm{C}$ until assessment. Fasting serum concentration of glucose, lipid profile, calcium and phosphate are determined by using enzymatic methods (Pars-Azmoon, Tehran, Iran) and an autoanalyzer (Selecta E; Vitalab, Holliston, Netherlands) on the same day of sampling. The intra- and interassay CVs for FSG and lipid concentrations are $<5 \%$.

Vitamin $\mathrm{D}$ status is determined by measuring the $25(\mathrm{OH})$ D concentrations, which is the major circulating form of vitamin D in blood (29). Mothers and cord blood serum concentrations of $25(\mathrm{OH}) \mathrm{D}$ are determined using a commercial enzyme-linked immunosorbent assay (ELISA) kit (EUROIMMUN) with high correlation of the measurement results with reference methods including LC-MS/MS and HPLC (High-performance liquid chromatography). The inter-assay coefficients of variation (CVs) ranged from 7 to 8.6- and intra-assay CVs ranged from 3.2 to $6.9 \%$, according to the manufacturer.

Fasting serum insulin is assayed by an ELISA kit (DiaPlus inc., Canada) with intra- and inter-assay
$\mathrm{CVs}$ are $4.9 \%$ and $8 \%$, respectively, based on manufacturer's data.

HOMA-IR which is used to evaluate insulin resistance and quantitative insulin sensitivity check index (QUICKI) which is used to evaluate insulin sensitivity are calculated based on suggested formulas(36).

Serum iPTH concentrations are measured by EUROIMMUN ELISA kit with intra- and inter-assay CVs 2.2\%-9.5\% and 9.5\%-11\%, respectively.

Serum hs-CRP is quantified using an ELISA kit (Pars-Azmoon, Tehran, Iran) with intra- and interassay CVs of $2.5 \%$ and $4.5 \%$, respectively.

Urinary concentrations of calcium and creatinine are determined using commercial kit and an autoanalyzer. In this study the proportion of urinary calcium to creatinine concentrations is used to adjust for the possible effects of urine dilution or concentration on changes of calcium concentrations in the spot urine sample.

Biomarkers of oxidative stress: Serum concentration of malondialdehyde (MDA) and total antioxidant capacity (TAC) are measured to determine oxidative stress. Serum MDA is measured using the Satoh method(37)with minor modifications by Neyestani et al (38).According to this method, firstly, proteins are precipitated using $20 \%$ trichloroacetic acid (TCA). Afterwards, the precipitate is washed with $0.05 \mathrm{M}$ sulfuric acid and then is reacted with thiobarbituric acid (TBA) at 90$100{ }^{\circ} \mathrm{C}$ for $30 \mathrm{~min}$. In the acidic environment $(\mathrm{pH}=2$ 3 ), one molecule of MDA binds to two TBA molecules to form a pink complex. Following extraction with n-butanol, absorbance is determined at $532 \mathrm{~nm}$ against the blank (n-butanol). Serum concentration of MDA was determined using a standard curve.

Serum, TAC, is evaluated using 2,2"-azinobis (3ethylbenzothiazoline- 6-sulfonate) (ABTS) as a reagent. ABTS under the influence of potassium persulfate is converted to ABTS cation radical $\left(\mathrm{ABTS}^{\circ+}\right.$ ) which is green-blue with maximum absorption at $415 \mathrm{~nm}, 645 \mathrm{~nm}, 734 \mathrm{~nm}$, and $815 \mathrm{~nm}$. Adding antioxidant solution to this in a given time attenuates color intensity depending on the antioxidant activity and concentration. Therefore, decolorization will be expressed as a percent of $\mathrm{ABTS}^{\circ}+$ inhibition based on difference of the primary and secondary absorbance divided by primary absorbance multiplied by 100 (39). Inhibition 
percentage is compared with the antioxidant activity of BSA as standard. Results are expressed as mmol/l of BSA.

Cell separation and culture for cytokine assays: Peripheral blood mononuclear cells (PBMCs) are separated and cultured with the method described elsewhere (40). Briefly, PBMCs are separated from fresh heparinized blood using Ficol gradient and then cultured in RPMI 1640 medium (Sigma-Aldrich, Inc., St. Louis, MO) supplemented with $100 \mathrm{U} / \mathrm{ml}$ penicillin $\mathrm{G}, 10 \mathrm{ng} / \mathrm{ml}$ streptomycin (both, SigmaAldrich, Inc.), $1 \%$ phytohemagglutinin, and 100 ng/ml lipopolysaccharide (Sigma) Human PBMC sare plated at a density of $2 \times 10^{6}$ cells/well in 12-well plates, which are kept for $24 \mathrm{~h}$ at $37^{\circ} \mathrm{C}$ in a $5 \%$ humidified $\mathrm{CO}_{2}$ incubator. Then, cytokines in the cell culture supernatants are measured using commercially available ELISA kits (all from Diaclone, Vienna, Austria) and a microplate reader (StatFax 3200; Awareness Technology, Inc., Palm City, FL, USA).

Adherence: The assessment of adherence to the determined vitamin $\mathrm{D}$ supplementation regimen is based on the pill counts and maternal self-report(41). Participants of each group are asked to return vitamin D pills that are not consumed for any reason each month. Compliance is calculated based on the following formula: (Number of pills dispensed number of pills remained)/(prescribed number of pills per day $\times$ number of days between 2 visits)(41).Participants who have $80 \%$ adherence to vitamin D supplements are considered to be compliant. Those who take lower than $50 \%$ of the prescribed vitamin D pills excluded from the study. Participants are reminded about the supplement regimen (vitamin D consumption) every week by telephone call.

Ethics: The present study is approved by the Ethics Committee of Shahid Beheshti University of Medical Sciences. This trial is registered on the RCT.gov (NCT03308487) and IRCT (IRCT2016090329675N1).

Safety Considerations: It has been shown that vitamin D supplementation can safely be utilized in pregnancy. Based on the results of previous trials prescribing 4000 IU of vitamin D3 per day (42) or circulating levels of $25(\mathrm{OH}) \mathrm{D}$ exceeding $300 \mathrm{nmol} / \mathrm{L}$ $(120 \mathrm{ng} / \mathrm{mL})$ do not cause hypercalciuria, as the first indicator of hypervitaminosis D (43). Furthermore the serum 25(OH) D level of $375 \mathrm{nmol} / \mathrm{L}(150 \mathrm{ng} / \mathrm{mL})$ and more, defined as hypervitaminosis $\mathrm{D}$ which could not be achieved by 1000 or $2000 \mathrm{IU} / \mathrm{d}$ vitamin D supplementation (42).

Statistical analysis: To check normal distribution of continuous variables the histogram and KolmogorovSmirnov test is applied. Data are presented as frequency distribution tables and numeric indices. Continuous data (normal in distribution) are presented as mean and standard deviation (SD) or median and interquartile range in the case of skewed distribution, and categorical data presented as frequency or percentage (\%). Paired Student's $t$-test (or Wilcoxon test) is used to compare baseline and endpoint measures (within group comparison). Independentsamples Student's $t$-test or Mann-Whitney test isapplied to compare data between groups when appropriate.

Correlations between variables are evaluated by using either Pearson ( $r$ ) (for data with normal distribution) or Spearman $\left(\mathrm{r}_{\mathrm{s}}\right)$ (for data with nonnormal distribution) correlations. The concentrations of inflammatory markers in cell-culture supernatants and lipid profile are compared by ANOVA for differences by vitamin $\mathrm{D}$ treatment group.

\section{Disc ussion}

A high prevalence $(60-80 \%)$ of vitamin D deficiency among Iranian pregnant women has been reported by several studies (3). Although some studies have reported that vitamin $\mathrm{D}$ supplementation during pregnancy may reduce the risk of maternal comorbidities attributed to vitamin $\mathrm{D}$ deficiency or insufficiency and help to improve neonatal outcomes (24-26), there is no general agreement on the optimal dose of vitamin $\mathrm{D}$ supplementation in pregnant women $(27,28)$. This lack of inconsistency could be attributed to the differences in study design, dose, type, and timing of vitamin D supplementation, and/or the subjects involved in the study with heterogeneous vitamin $\mathrm{D}$ status, and/or methodological differences. It is noteworthy that the effectiveness of the current recommendation of 1000 IU vitamin $\mathrm{D} / \mathrm{d}$ during pregnancy made by the $\mathrm{Ir}$ $\mathrm{MOH}$ has not been documented by far. The current study attempts to evaluate the effectiveness of two different doses of vitamin $\mathrm{D}$ by using a wide spectrum of maternal and neonatal biomarkers. The results of this study could be potentially valuable for further development of guidelines and policy-making on vitamin D supplementation during pregnancy. 


\section{Strengths of the study}

The advantages of the present study are as follows: 1) the randomized clinical design,2) initiating vitamin D supplementation early in pregnancy until delivery, 3) evaluating the confounding factors (diet, physical activity level, sun exposure), 4) Assessing the metabolic, oxidative stress and inflammatory markers in maternal and cord blood samples.

The results of the present study will help clinicians regarding administration an optimal dose of vitamin D supplementation during pregnancy and also will help guide the public health policymaker to revise the currently used vitamin D supplementation protocol among pregnant women.

\section{Financial disclosure}

The authors declared no financial interest.

\section{References}

1.Palacios C, Gonzalez L. Is vitamin D deficiency a major global public health problem? The Journal of steroid biochemistry and molecular biology. 2014 Oct;144 Pt A:138-45.

2.Saraf R, Morton SM, Camargo CA, Jr., Grant CC. Global summary of maternal and newborn vitamin D status - a systematic review. Maternal \& child nutrition. 2016 Oct;12(4):647-68.

3.Pirdehghan A, Vakili M, Dehghan R, Zare F. High prevalence of Vitamin D deficiency and adverse pregnancy outcomes in Yazd, a central province of Iran. Journal of Reproduction and Infertility. 2016;17(1):348.

4.Abbasian M, Chaman R, Amiri M, Ajami ME, JafariKoshki T, Rohani H, et al. Vitamin D Deficiency in Pregnant Women and Their Neonates. Glob J Health Sci. 2016 Sep 1;8(9):54008.

5.Kelishadi R, Sharifi-Ghazvini F, Poursafa P, Mehrabian F, Farajian $\mathrm{S}$, Yousefy $\mathrm{H}$, et al. Determinants of hypovitaminosis $\mathrm{D}$ in pregnant women and their newborns in a sunny region. International Journal of Endocrinology. 2013;2013.

6.Schneuer FJ, Roberts CL, Guilbert C, Simpson JM, Algert CS, Khambalia AZ, et al. Effects of maternal serum 25hydroxyvitamin $\mathrm{D}$ concentrations in the first trimester on subsequent pregnancy outcomes in an Australian population. Am J Clin Nutr. 2014 Feb;99(2):287-95.

7.Cashman KD, Dowling KG, Skrabakova Z, GonzalezGross M, Valtuena J, De Henauw S, et al. Vitamin D deficiency in Europe: pandemic? Am J Clin Nutr. 2016 Apr;103(4):1033-44.

8.Olmos-Ortiz A, Avila E, Durand-Carbajal M, Diaz L. Regulation of calcitriol biosynthesis and activity: focus on gestational vitamin D deficiency and adverse pregnancy outcomes. Nutrients. 2015 Jan 09;7(1):44380. PubMed PMID: 25584965.
9.Ji JL, Muyayalo KP, Zhang YH, Hu XH, Liao AH. Immunological function of vitamin $\mathrm{D}$ during human pregnancy. American Journal of Reproductive Immunology. 2017;78(2).

10.Wagner CL, Wei W, Shary JR, Ebeling MD, GarrettMayer E, Taylor SN, et al. Preventing Health Disparities during Pregnancy through Vitamin D (VitD) Supplementation: Preliminary Results of a Randomized Controlled Trial. Faseb Journal. 2017 Apr;31.

11.Moon RJ, Harvey NC, Cooper C. ENDOCRINOLOGY IN PREGNANCY: Influence of maternal vitamin D status on obstetric outcomes and the fetal skeleton. European Journal of Endocrinology. 2015;173(2):R69R83.

12.Chen X, Rao SQ, Gao BH, Jiang ZQ. Effect of early vitamin $\mathrm{D}$ supplementation on asthma and the possible mechanisms. Genetics and Molecular Research. 2015;14(4):14136-43.

13.Hollis BW, Wagner CL. Vitamin D supplementation during pregnancy: Improvements in birth outcomes and complications through direct genomic alteration. Molecular and Cellular Endocrinology. 2017;453:11330.

14.Liu NQ, Kaplan AT, Lagishetty V, Ouyang YB, Ouyang Y, Simmons CF, et al. Vitamin D and the regulation of placental inflammation. Journal of Immunology. 2011;186(10):5968-74.

15.Racicot K, Kwon JY, Aldo P, Silasi M, Mor G. Understanding the complexity of the immune system during pregnancy. American journal of reproductive immunology (New York, NY : 1989). 2014 Aug;72(2):107-16.

16.Noyola-Martinez N, Diaz L, Avila E, Halhali A, Larrea F, Barrera D. Calcitriol downregulates TNF-alpha and IL6 expression in cultured placental cells from preeclamptic women. Cytokine. 2013 Jan;61(1):245-50.

17.Ota K, Dambaeva S, Kim MW, Han AR, Fukui A, Gilman-Sachs A, et al. 1,25-Dihydroxy-vitamin D3 regulates NK-cell cytotoxicity, cytokine secretion, and degranulation in women with recurrent pregnancy losses. Eur J Immunol. 2015 Nov;45(11):3188-99.

18.Tamblyn JA, Hewison M, Wagner CL, Bulmer JN, Kilby MD. Immunological role of vitamin $\mathrm{D}$ at the maternalfetal interface. J Endocrinol. 2015 Mar;224(3):R107-21.

19.Tamblyn JA, Susarla R, Jenkinson C, Jeffery LE, Ohizua $\mathrm{O}$, Chun RF, et al. Dysregulation of maternal and placental vitamin $\mathrm{D}$ metabolism in preeclampsia. Placenta. 2017;50:70-7.

20.Liu NQ, Ouyang Y, Bulut Y, Lagishetty V, Chan SY, Hollis BW, et al. Dietary vitamin D restriction in pregnant female mice is associated with maternal hypertension and altered placental and fetal development. Endocrinology. 2013;154(7):2270-80.

21.Barrera D, Diaz L, Noyola-Martinez N, Halhali A. Vitamin D and Inflammatory Cytokines in Healthy and Preeclamptic Pregnancies. Nutrients. 2015 Aug 04;7(8):6465-90. 
22.Ross AC, Manson JE, Abrams SA, Aloia JF, Brannon PM, Clinton SK, et al. The 2011 Dietary Reference Intakes for Calcium and Vitamin D: what dietetics practitioners need to know. J Am Diet Assoc. 2011 Apr;111(4):524-7.

23.Hollis BW, Johnson D, Hulsey TC, Ebeling M, Wagner CL. Erratum: Vitamin D supplementation during pregnancy: Double-blind, randomized clinical trial of safety and effectiveness (Journal of Bone and Mineral Research (2011) 26 (2341-2357) DOI: 10 DOI: 10.1002/jbmr.463). Journal of Bone and Mineral Research. 2011;26(12):3001.

24.Sablok A, Batra A, Thariani K, Batra A, Bharti R, Aggarwal AR, et al. Supplementation of Vitamin D in pregnancy and its correlation with feto-maternal outcome. Clinical Endocrinology. 2015;83(4):536-41.

25.Rodda CP, Benson JE, Vincent AJ, Whitehead CL, Polykov A, Vollenhoven B. Maternal Vitamin D supplementation during pregnancy prevents Vitamin D deficiency in the newborn: An open-label randomized controlled trial. Clinical Endocrinology. 2015;83(3):363-8.

26.Aghajafari F, Field CJ, Kaplan BJ, Maggiore JA, O'Beirne M, Hanley DA, et al. The High Prevalence of Vitamin D Insufficiency in Cord Blood in Calgary, Alberta (APrON-D Study). Journal of Obstetrics and Gynaecology Canada. 2017;39(5):347-53.e1.

27.Figueiredo AC, Cocate PG, Adegboye AR, Franco-Sena $\mathrm{AB}$, Farias DR, de Castro MB, et al. Changes in plasma concentrations of 25-hydroxyvitamin $\mathrm{D}$ and 1,25dihydroxyvitamin D during pregnancy: a Brazilian cohort. Eur J Nutr. 2017 Mar 28. PubMed PMID: 28353072. Epub 2017/03/30. eng.

28. Karras SN, Anagnostis P, Naughton D, Annweiler C, Petroczi A, Goulis DG. Vitamin D during pregnancy: Why observational studies suggest deficiency and interventional studies show no improvement in clinical outcomes? A narrative review. Journal of Endocrinological Investigation. 2015;38(12):1265-75.

29.Rosen CJ, Abrams SA, Aloia JF, Brannon PM, Clinton SK, Durazo-Arvizu RA, et al. IOM committee members respond to Endocrine Society vitamin D guideline. J Clin Endocrinol Metab. 2012 Apr;97(4):1146-52. PubMed PMID: 22442278. Pubmed Central PMCID: PMC5393439. Epub 2012/03/24. eng.

30. Weinert LS. International Association of Diabetes and Pregnancy Study Groups recommendations on the diagnosis and classification of hyperglycemia in pregnancy: comment to the International Association of Diabetes and Pregnancy Study Groups Consensus Panel. Diabetes Care. 2010 Jul;33(7):e97; author reply e8. PubMed PMID: 20587717. Epub 2010/07/01. eng.

31.Preterm Birth: Causes, Consequences, and Prevention. Behrman RE, Butler AS, editors. Washington DC: National Academy of Sciences.; 2007.
32.Bodnar LM, Catov JM, Zmuda JM, Cooper ME, Parrott MS, Roberts JM, et al. Maternal serum 25hydroxyvitamin $\mathrm{D}$ concentrations are associated with small-for-gestational age births in white women. Journal of Nutrition. 2010;140(5):999-1006.

33.Ford HB, Schust DJ. Recurrent pregnancy loss: etiology, diagnosis, and therapy. Reviews in obstetrics \& gynecology. 2009 Spring;2(2):76-83.

34.Uzan J, Carbonnel M, Piconne O, Asmar R, Ayoubi JM. Pre-eclampsia: pathophysiology, diagnosis, and management. Vascular health and risk management. 2011;7:467-74.

35.Brustad M, Alsaker E, Engelsen O, Aksnes L, Lund E. Vitamin D status of middle-aged women at 65-71 degrees $\mathrm{N}$ in relation to dietary intake and exposure to ultraviolet radiation. Public Health Nutr. 2004 Apr;7(2):327-35.

36.Matthews DR, Hosker JP, Rudenski AS, Naylor BA, Treacher DF, Turner RC. Homeostasis model assessment: insulin resistance and beta-cell function from fasting plasma glucose and insulin concentrations in man. Diabetologia. 1985 Jul;28(7):412-9.

37.Satoh K. Serum lipid peroxide in cerebrovascular disorders determined by a new colorimetric method. Clinica chimica acta; international journal of clinical chemistry. 1978 Nov 15;90(1):37-43.

38.Neyestani TR, Shariatzadeh N, Gharavi A, Kalayi A, Khalaji N. Physiological dose of lycopene suppressed oxidative stress and enhanced serum levels of immunoglobulin $M$ in patients with Type 2 diabetes mellitus: a possible role in the prevention of long-term complications. J Endocrinol Invest. 2007 Nov;30(10):833-8.

39.Rice-Evans C, Miller NJ. Total antioxidant status in plasma and body fluids. Methods in enzymology. 1994;234:279-93.

40.Neyestani TR, Gharavi A, Kalayi A. Selective effects of tea extract and its phenolic compounds on human peripheral blood mononuclear cell cytokine secretions. Int J Food Sci Nutr. 2009;60 Suppl 1:79-88.

41.Appelgren KE, Nietert PJ, Hulsey TC, Hollis BW, Wagner CL. Analyzing Adherence to Prenatal Supplement: Does Pill Count Measure Up? International Journal of Endocrinology. 2010.

42.Hollis BW, Johnson D, Hulsey TC, Ebeling M, Wagner CL. Vitamin D supplementation during pregnancy: Double-blind, randomized clinical trial of safety and effectiveness. Journal of Bone and Mineral Research. 2011;26(10):2341-57.

43.Heaney RP, Davies KM, Chen TC, Holick MF, BargerLux MJ. Human serum 25-hydroxycholecalciferol response to extended oral dosing with cholecalciferol. Am J Clin Nutr. 2003 Jan;77(1):204-10. 